\title{
Percutaneous peritoneal dialysis catheter implantation with no break-in period: a viable option for patients requiring unplanned urgent-start peritoneal dialysis
}

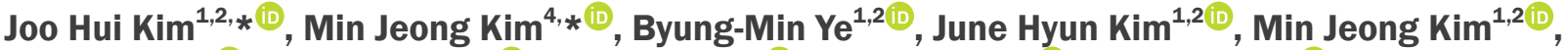

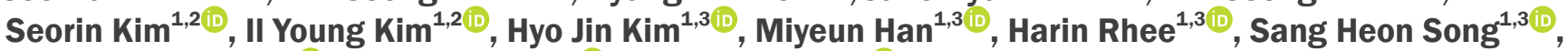 \\ Eun Young Seong ${ }^{1,3 \mathbb{C}}$, Soo Bong Lee ${ }^{1,2 \mathbb{E}}$, Dong Won Lee $e^{1,2 \mathbb{E}}$ \\ 'Department of Internal Medicine, Pusan National University School of Medicine, Yangsan, Republic of Korea \\ ${ }^{2}$ Division of Nephrology, Department of Internal Medicine, Pusan National University Yangsan Hospital, Yangsan, Republic of Korea \\ ${ }^{3}$ Division of Nephrology, Pusan National University Hospital, Busan, Republic of Korea \\ ${ }^{4}$ Department of Internal Medicine, Gimhae Bokum Hospital, Gimhae, Republic of Korea
}

Background: Urgent-start peritoneal dialysis (PD) is applied to patients who need PD within two weeks but are able to wait for more than 48 hours before starting PD. To evaluate the usefulness of percutaneous PD catheter insertion in urgent-start PD, we reviewed the clinical outcomes of percutaneous catheter insertion with immediate start PD and surgical insertion with longer break-in time in Pusan National University Hospital.

Methods: This study included 177 patients who underwent urgent-start PD. Based on the PD catheter insertion techniques, the patients with urgent-start PD were divided into percutaneous $(n=103)$ and surgical $(n=74)$ groups. For the percutaneous group, a modified Seldinger percutaneous catheter insertion with immediate initiation of continuous ambulatory PD was performed by nephrologists.

Results: The percutaneous group showed higher serum urea nitrogen, creatinine, and lower serum albumin compared with the surgical group $(P<0.05)$. Ninety-day infectious and mechanical complications showed no significant differences between the two groups. Ninety-day peritonitis in the percutaneous group was $9.7 \%$ compared to $5.4 \%$ in the surgical group ( $P=$ not significant [NS]). Major leakage was 3.9\% in the percutaneous group compared to $1.4 \%$ in the surgical group $(P=N S$ ). Overall infectious and mechanical complication-free survival was not significantly different between the two groups. The percutaneous group and surgical group showed no statistical difference with respect to catheter survival over the entire observation period ( $P=\mathrm{NS}$ ).

Conclusion: This study suggests that urgent-start PD can be applied safely with percutaneous catheter insertion by nephrologists with no break-in period.

Keywords: Catheters, Complications, Peritoneal dialysis, Urgent-start

Received January 10, 2020; Revised May 6, 2020; Accepted May 8, 2020

Editor: Chan-Duck Kim, Kyungpook National University, Daegu, Republic of Korea

Correspondence: Dong Won Lee

Division of Nephrology, Department of Internal Medicine, Pusan National University Yangsan Hospital, 20 Geumo-ro, Mulgeum-eup, Yangsan 50612, Republic of Korea. E-mail: dongwonlee@pusan.ac.kr

*Joo Hui Kim and Min Jeong Kim contributed equally to this work.

Copyright (c) 2020 by The Korean Society of Nephrology

(a) This is an open-access article distributed under the terms of the Creative Commons Attribution Non-Commercial License (http://creativecommons.org/ licenses/by-nc-nd/4.0/), which permits unrestricted non-commercial use, distribution, and reproduction in any medium, provided the original work is properly cited. 


\section{Introduction}

A large number of patients with chronic kidney disease (CKD) encounter dialysis without mature hemodialysis (HD) vascular access. More than $80 \%$ of incident HD patients start dialysis treatment with a central venous catheter (CVC) in the US and Canada [1]. A considerable number of unplanned end stage renal disease (ESRD) patients, although suitable for peritoneal dialysis (PD), are still started with temporary HD via tunneled CVC. Several factors for the underutilization of PD in unplanned situations are increased in elderly ESRD patients due to frailty as an underlying condition and the proliferation of HD facilities for treatment. Moreover, disillusionment among physicians about long term maintenance of $\mathrm{PD}$ for their patients, and easy accessibility to CVC, also contribute to the underuse of PD [2], even though the initiation of HD with CVC has several life-threatening risks including CVC-related bacteremia, septicemia [3,4], and/or deepseated infections [5]. In almost all countries, patients starting PD have unfavorable baseline characteristics that may translate to worse survival; however, the survival rate of patients starting PD has gradually and significantly improved due to advancements in standard PD patient care $[6,7]$.

Unless there is a need for emergent dialysis due to refractory hypertension, pulmonary edema, hyperkalemia, or uremic pericarditis, a recent study has suggested that an urgent-start PD program could be a good option for patients with no access to dialysis [8]. The definition of urgent-start PD is the initiation of PD therapy within two weeks after PD catheter insertion in an ESRD patient requiring dialysis in less than two weeks who is not predicted to require long-term dialysis [9]. In this urgent setting, the percutaneous PD catheter insertion technique with short or lack of a break-in period would be more suitable than the surgical method that requires a longer breakin period for preventing early mechanical complications such as pericatheter leakage. The 2005 European Best Practice Guidelines for peritoneal access recommends at least a two week break-in period to avoid early leakage [10]. In 2010, the International Society for Peritoneal Dialysis (ISPD) recommended at least a two-week interval between catheter insertion and the initiation of PD [11]. Improper peritoneal healing and increased rate of early pericatheter leakage during the short break-in period were the main concerns; however, several recent reports showed favorable outcomes with the initiation of PD within two-weeks after catheter insertion by the percutaneous method [9] or by surgical method [12].

Recently, Alkatheeri et al [13] reported the Canadian experience with an urgent-start PD program using the percutaneous or surgical catheter insertion methods. However, little is known about the comparison of percutaneous and surgical methods in the urgent PD setting with a large number of patients during a longer observation period. For more than 15 years, Korean nephrologists have performed immediate start PD with no breakin period using a modified Seldinger percutaneous PD catheter insertion method [14]. The aim of this study was to compare infectious and mechanical complications between percutaneous PD catheter insertion with no breakin period and surgical insertion with longer break-in period and catheter survival in the urgent setting.

\section{Methods}

From January 1, 2010 to December 31, 2017, 205 patients who underwent urgent PD catheter insertion were retrospectively reviewed. Regardless of bridging HD via CVC as emergent-start dialysis, the initiation of PD within two weeks after catheter insertion was basically regarded as urgent-start PD. The initiation of PD two weeks after catheter insertion was regarded as elective or non-urgent PD. Exclusion criteria were $<18$ years of age, elective PD, loss of operation record, and/or switching modality from maintenance HD to PD. The remaining 177 patients were divided into either the percutaneous or surgical group according to catheter insertion technique (Fig. 1).

The PD catheter used was a straight, double-cuffed Tenckhoff catheter with a straight or swan-neck intraperitoneal segment. In the percutaneous group, the catheters were inserted under local anesthesia by two nephrologists. A prophylactic antibiotic (cefazolin $1 \mathrm{~g}$, intravenous) was administered one hour before the procedure. A paramedian vertical $3-5 \mathrm{~cm}$ skin incision was made at $2 \mathrm{~cm}$ inferior to and $1 \mathrm{~cm}$ left lateral of the umbilicus. The subcutaneous tissue was dissected down to the anterior rectus sheath. A 16-gauge angiocatheter was inserted in a perpendicular direction into the abdomen and the needle was removed, leaving the plastic tube. Two liters of dialysis (1.5\% glucose) solution were infused into the 


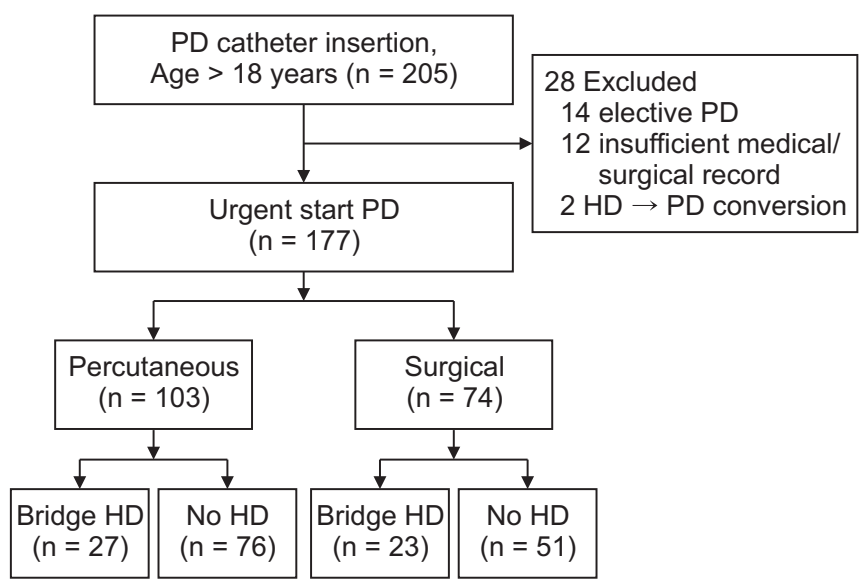

Figure 1. Patient enrollment in urgent-start peritoneal dialysis (PD).

$\mathrm{HD}$, hemodialysis.

peritoneal cavity through the plastic tube. A guidewire was inserted through the plastic tube, which was then removed. Tapered dilators (Shin dilators) with different external diameters were inserted over the guidewire and removed in the order of diameter. After both the guidewire and the dilator were removed, a Tenckhoff catheter with a stylet was introduced into the deep pelvis, aiming 10 degrees off the perpendicular toward the coccyx or pre-sacral fossa and through the intramuscular tract that had been made by the dilators. The stylet was removed, leaving the deep cuff on the anterior rectus sheath. While the patient tensed the abdominal wall, the deep cuff was pushed into the rectus muscle immediately below the anterior rectus sheath. The catheter was secured by pursestring sutures around the deep cuff. A subcutaneous tunnel was created using a piercing trocar from the subcutaneous portion to the stab wound of the skin exit site. The subcutaneous cuff was placed $2 \mathrm{~cm}$ from the skin exit. The Tenckhoff catheter was connected to the dialysis tubing and the fluid in the abdomen was drained during the procedure. Immediately after insertion, the catheter was irrigated with $500 \mathrm{~mL}$ dialysis solution three times. After irrigation, PD was initiated with no break-in period with $500 \mathrm{~mL}$ dialysis (1.5\% glucose) solution four times a day. All patients stayed in a supine position with minimal ambulation during the first three days. PD was continued with step-wise increasing volume of dialysis solution and four, $2 \mathrm{~L}$ exchanges per day were performed starting 10 to 14 days after catheter insertion [14].

In the surgical group, the same general antibiotic pro- phylaxis and catheter were used as in the percutaneous group. Laparoscopic or open insertion techniques were used by the general surgeons. After a 7 day break-in period, PD was started with gradual increasing dialysate volume.

The first dressing interval after operation was 4 to 5 days in both groups, after which daily care of the exit site was applied. Povidone iodine or $2 \%$ chlorhexidine were used as disinfectants. Mupirocin as a prophylactic topical antibiotic was used.

Baseline characteristics such as age, weight $(\mathrm{kg})$, height $(\mathrm{cm})$, body mass index (BMI, $\left.\mathrm{kg} / \mathrm{m}^{2}\right)$, and initial laboratory findings such as hemoglobin $(\mathrm{Hb}, \mathrm{g} / \mathrm{dL})$, albumin $(\mathrm{g} /$ $\mathrm{dL}$ ), blood urea nitrogen (BUN, $\mathrm{mg} / \mathrm{dL}$ ), serum creatinine (SCr, mg/dL), estimated glomerular filtration rate (eGFR, MDRD, $\mathrm{mL} / \mathrm{min} / 1.73 \mathrm{~m}^{2}$ ) and the causes of CKD were obtained from patient medical records. Follow-up days were calculated from the date of catheter insertion to the date of the last clinic visit, conversion to HD, kidney transplantation (KT), or death by any causes.

Any events of infectious or mechanical complications were checked. Infectious complications included peritonitis, exit site infection, and tunnel infection. Infectious complication definitions were consistent with the ISPD guidelines [15]. Mechanical complications included pericatheter leakage, catheter migration requiring intervention, diminished outflow requiring catheter intervention or switch of the dialysis modality, hemorrhage resulting in temporary cessation of PD, bowel perforation, and hernia.

Continuous parameters were compared using independent $t$ tests and are reported as the mean \pm standard deviation. Categorical parameters were compared using the chi-square test and are reported as a proportion. Statistical differences were considered significant at a $P$ value of $<0.05$. Infectious and mechanical complication-free survival and catheter survival were analyzed using the Kaplan-Meier method. Complication-free survival (days) was calculated from the date of catheter insertion to the first event or any infectious or mechanical complications. Catheter failure was defined as catheter removal due to infectious or mechanical complications. Cases of KT and death were censored in catheter failure. Catheter survival (days) was calculated from the date of catheter insertion to the date of catheter failure and the re-inserted catheter was counted as an additional catheter. As a result, a total 
Table 1. Baseline patient clinical characteristics

\begin{tabular}{lccr}
\hline \multicolumn{1}{c}{ Characteristic } & $\begin{array}{c}\text { Percutaneous } \\
\text { group }(\mathrm{n}=103)\end{array}$ & $\begin{array}{c}\text { Surgical } \\
\text { group }(\mathrm{n}=74)\end{array}$ & $P$ value \\
\hline Age $(\mathrm{yr})$ & $48.0 \pm 12.5$ & $48.8 \pm 12.8$ & 0.681 \\
Weight $(\mathrm{kg})$ & $66.2 \pm 11.1$ & $64.1 \pm 11.62$ & 0.220 \\
Height $(\mathrm{cm})$ & $166.0 \pm 12.8$ & $165.1 \pm 7.8$ & 0.562 \\
BMI $\left(\mathrm{kg} / \mathrm{m}^{2}\right)$ & $24.7 \pm 10.6$ & $23.4 \pm 3.3$ & 0.310 \\
Hemoglobin $(\mathrm{g} / \mathrm{dL})$ & $9.3 \pm 1.5$ & $9.9 \pm 1.3$ & 0.002 \\
Albumin $(\mathrm{g} / \mathrm{dL})$ & $3.3 \pm 0.6$ & $3.6 \pm 0.6$ & 0.009 \\
BUN $(\mathrm{mg} / \mathrm{dL})$ & $93.9 \pm 38.8$ & $63.4 \pm 25.7$ & $<0.001$ \\
SCr $(\mathrm{mg} / \mathrm{dL})$ & $10.1 \pm 3.9$ & $7.7 \pm 2.1$ & $<0.001$ \\
eGFR $\left(\mathrm{mL} / \mathrm{min} / 1.73 \mathrm{~m}^{2}\right)$ & $6.4 \pm 2.8$ & $7.7 \pm 2.5$ & 0.002 \\
Causes of CKD & & & 0.052 \\
$\quad$ Diabetes & $48(46.6)$ & $35(47.3)$ & \\
Hypertension & $22(21.4)$ & $12(16.2)$ & \\
Glomerulonephritis & $20(19.4)$ & $25(33.8)$ & \\
Unknown & $10(9.7)$ & $1(1.4)$ & \\
Other ${ }^{\mathrm{a}}$ & $3(2.9)$ & $1(1.4)$ & \\
Follow-up period $(\mathrm{d})$ & $1,561 \pm 1,214$ & $1,675 \pm 1,349$ & 0.557 \\
\hline
\end{tabular}

Data are presented as mean \pm standard deviation or number (\%).

$\mathrm{BMI}$, body mass index; BUN, blood urea nitrogen; CKD, chronic kidney disease; eGFR, estimated glomerular filtration rate by MDRD (Modification of Diet in Renal Disease Study) equation; SCr, serum creatinine.

${ }^{a}$ Autosomal dominant polycystic kidney disease and reflux nephropathy.

of 186 catheters were analyzed for catheter survival. Univariate and multivariate Cox proportional hazards models were used to determine the independent risk factors for catheter failure. We performed noninferiority tests to investigate if the percutaneous group was superior to the surgical group for catheter survival. The noninferiority margin was selected as 15 percentage points, and noninferiority would be claimed if the lower limit of the twosided $95 \%$ confidential interval (CI) for the risk difference was not below -15 percent points. All analyses were performed using the IBM SPSS Statistics version 21.0 (IBM Corp., Armonk, NY, USA). This study protocol was approved by the Pusan National University Yangsan Hospital Institutional Review Board (IRB no. 05-2016-114, No. 1609-001-045), and informed consent was waived.

\section{Results}

A total of 177 patients started urgent PD from January 1, 2010 to December 31, 2017 in either the percutaneous group $(n=103)$ or the surgical group $(n=74)$. The mean follow-up duration was $1,561 \pm 1,214$ days in the percutaneous group and $1,675 \pm 1,349$ days in the surgical group
Table 2. Complications within 90 days after catheter insertion

\begin{tabular}{lccc}
\hline & $\begin{array}{c}\text { Percutaneous } \\
\text { group }(\mathrm{n}=103)\end{array}$ & $\begin{array}{c}\text { Surgical } \\
\text { group }(\mathrm{n}=74)\end{array}$ & $P$ value \\
\hline Infectious & & & \\
$\quad$ Peritonitis & $10(9.7)$ & $4(5.4)$ & 0.401 \\
Exit site infection & $0(0.0)$ & $1(1.4)$ & 0.418 \\
Tunnel infection & $0(0.0)$ & $0(0.0)$ & \\
Mechanical & & & \\
Major leakage & $4(3.9)$ & $1(1.4)$ & 0.402 \\
Migration & $3(2.9)$ & $1(1.4)$ & 0.641 \\
Diminished outflow & $3(2.9)$ & $0(0.0)$ & 0.266 \\
Hemorrhage & $0(0.0)$ & $1(1.4)$ & 0.418 \\
Bowel perforation & $0(0.0)$ & $0(0.0)$ & \\
Hernia & $0(0.0)$ & $1(1.4)$ & 0.418 \\
\hline
\end{tabular}

Data are presented as number (\%).

( $P=$ not significant $[\mathrm{NS}]$ ).

There were no differences in age, weight, height, or BMI between the two groups. The percutaneous group showed significantly lower $\mathrm{Hb}$, albumin, higher BUN, SCr, and lower eGFR than the surgical group (Table 1). The percutaneous group showed an immediate start of PD with no break-in period according to the protocol. The surgical group had delayed start of PD (break-in period, $10.9 \pm 2.05$ days; range, 8 to 14 days; $P<0.01$ ). The incidence of bridging HD prior to PD was $26.2 \%(27 / 103)$ in the percutaneous group and $31.1 \%(23 / 74)$ in the surgical group ( $P=$ NS). The length of hospitalization was $12.7 \pm 7.7$ days in the percutaneous group and $14.5 \pm 9.0$ days in the surgical group $(P<0.01)$.

The 90-day infectious complications showed no significant differences between the two groups (Table 2). The 90day peritonitis in the percutaneous group was $9.7 \%(10 / 103)$ compared to $5.4 \%(4 / 74)$ in the surgical group $(P=N S)$. There were no exit site or tunnel infections within 90 days in the percutaneous group. The 90-day mechanical complications showed no significant differences between the two groups (Table 2). Major leakage with temporary cessation of dialysate dwelling was $3.9 \%(4 / 103)$ in the percutaneous group, and $1.4 \%(1 / 74)$ in the surgical group $(P=$ NS). Only one occurrence of hemorrhage with rectus muscle bleeding was identified in the surgical group, which was treated with embolization of the inferior epigastric artery. Within the follow-up period, the infectious and mechanical complication-free survival in both groups were not significantly different based on KaplanMeier analysis (Fig. 2, 3). 


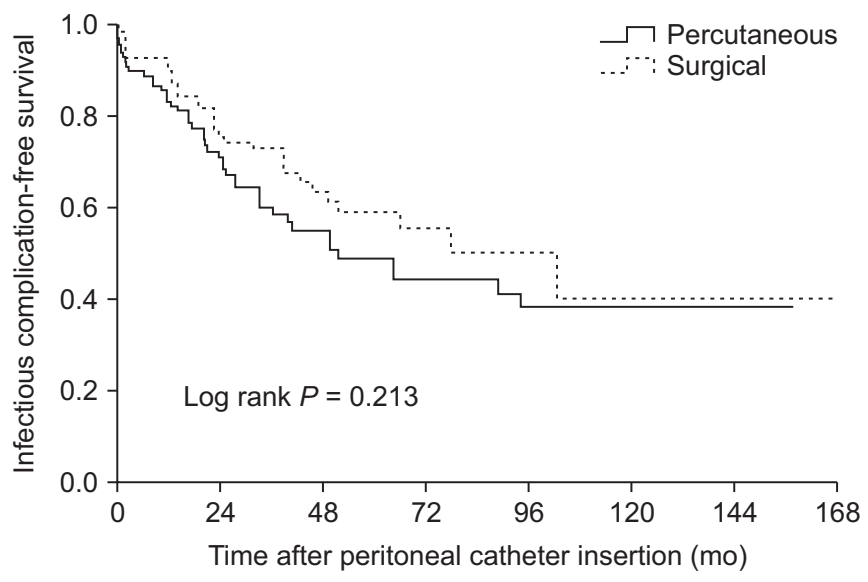

Figure 2. Infectious complication-free survival using the KaplanMeier survival analysis. The percutaneous and surgical groups showed no statistical difference with respect to overall infectious complication-free survival during the entire observation period.

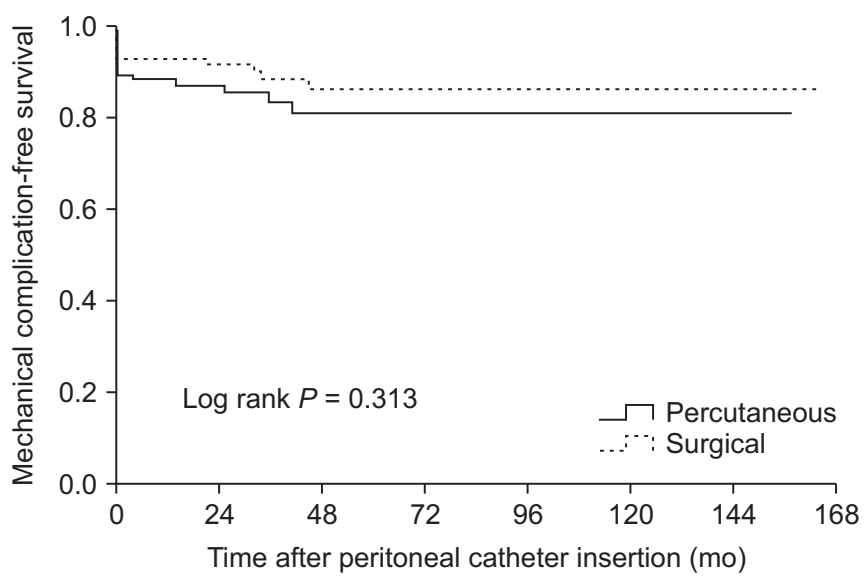

Figure 3. Mechanical complication-free survival using the KaplanMeier survival analysis. The percutaneous and surgical groups showed no statistical difference with respect to overall mechanical complication-free survival during the entire observation period.

Catheter reinsertion rate was $4.9 \%(5 / 103)$ in the percutaneous group and $5.4 \%(4 / 74)$ in the surgical group. In the percutaneous group, catheter reinsertion was completed in three cases within two weeks after catheter insertion. The causes of early reinsertion in the percutaneous group included one major leakage, one catheter migration, and one omental wrapping. The causes of reinsertion in the percutaneous group after two weeks of operation were one recurrent $\mathrm{PD}$ peritonitis and one external cuff exposure due to extreme truncal obesity. No early catheter reinsertions occurred in the surgical group. The causes of catheter reinsertion in the surgical group during the entire follow-up period included one refrac-

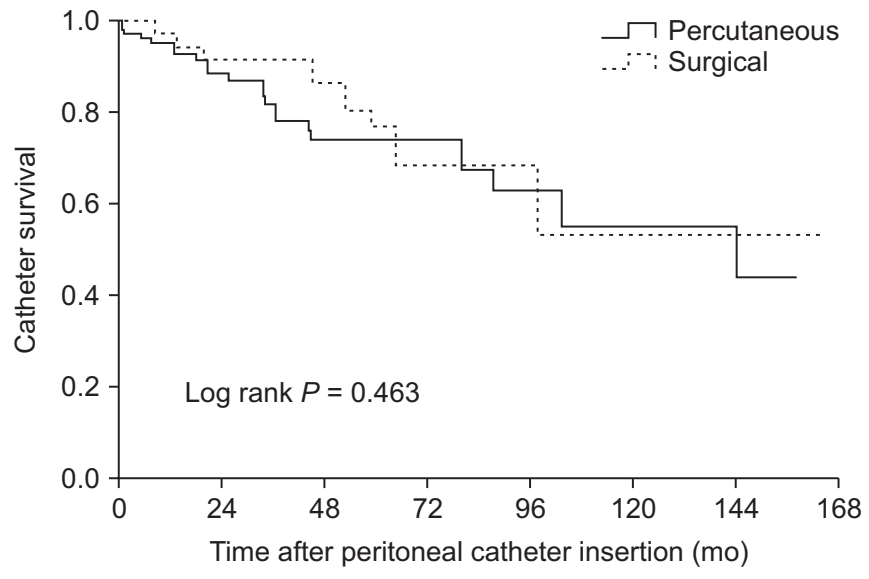

Figure 4. Catheter survival using the Kaplan-Meier survival analysis. The percutaneous and surgical groups showed no statistical difference with respect to catheter survival during the entire observation period.

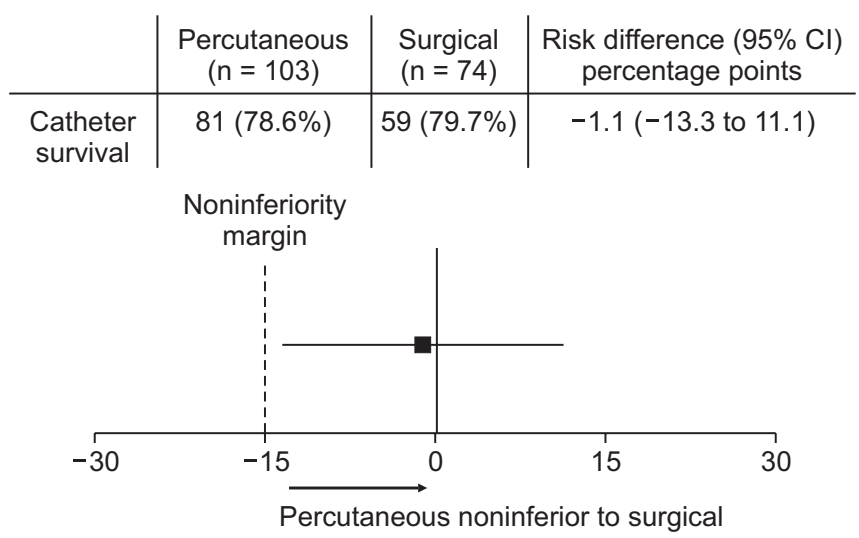

Figure 5. Noninferiority test for catheter survival. The percutaneous group was not inferior to the surgical group for catheter survival (risk difference, -1.1 percent points; 95\% confidential interval $[\mathrm{Cl}]$, -13.3 to 11.1 ; noninferiority margin, -15 percent points).

tory exit site infection, one refractory tunnel infection, and two recurrent peritonitis.

When counting reinserted catheters as an additional catheter, a total of 186 catheter insertions were conducted. There was no significant difference in catheter survival between the percutaneous and surgical groups (log rank test, $P=0.463$; Fig. 4 ). The catheter survival rate was $78.6 \%(81 / 103)$ in the percutaneous group and $79.7 \%$ $(59 / 74)$ in the surgical group. In the noninferiority test, the percutaneous group was not inferior to the surgical group for catheter survival (risk difference, -1.1 percent points; $95 \% \mathrm{CI},-13.3$ to 11.1 ; noninferiority margin, -15 percent points; Fig. 5). We also performed a Cox regres- 
Table 3. Univariate and multivariate Cox regression analyses for variables associated with catheter failure in patients

\begin{tabular}{|c|c|c|c|c|}
\hline & \multicolumn{2}{|c|}{ Univariate } & \multicolumn{2}{|c|}{ Multivariate } \\
\hline & $\mathrm{HR}(95 \% \mathrm{Cl})$ & $P$ value & $\mathrm{HR}(95 \% \mathrm{Cl})$ & $P$ value \\
\hline $\begin{array}{l}\text { PD catheter insertion method, percutaneous } \\
\text { (vs. surgical) }\end{array}$ & $0.91(0.47-1.78)$ & 0.790 & $0.90(0.43-1.92)$ & 0.794 \\
\hline Age (per 1-year increase) & $1.02(0.99-1.05)$ & 0.157 & $1.02(0.99-1.05)$ & 0.274 \\
\hline Sex (vs. female) & $0.81(0.43-1.55)$ & 0.530 & $0.91(0.47-1.79)$ & 0.790 \\
\hline BMI (per $1 \mathrm{~kg} / \mathrm{m}^{2}$ increase) & $1.00(0.90-1.12)$ & 0.997 & $1.00(0.88-1.14)$ & 0.979 \\
\hline Hemoglobin (per $1 \mathrm{~g} / \mathrm{dL}$ increase) & $0.98(0.81-1.17)$ & 0.782 & $0.93(0.74-1.17)$ & 0.240 \\
\hline Albumin $(1 \mathrm{~g} / \mathrm{dL}$ increase $)$ & $0.75(0.45-1.27)$ & 0.286 & $0.71(0.41-1.25)$ & 0.232 \\
\hline eGFR (per $1 \mathrm{~mL} / \mathrm{min} / 1.73 \mathrm{~m}^{2}$ increase) & $1.06(0.91-1.23)$ & 0.464 & $1.08(0.91-1.28)$ & 0.360 \\
\hline Diabetes mellitus (vs. no diabetes mellitus) & $1.27(0.66-2.47)$ & 0.479 & $0.58(0.17-1.99)$ & 0.386 \\
\hline Hypertension (vs. no hypertension) & $0.78(0.32-1.87)$ & 0.574 & $0.58(0.17-1.98)$ & 0.386 \\
\hline Glomerulonephritis (vs. no glomerulonephritis) & $0.46(0.16-1.31)$ & 0.146 & $0.39(0.11-1.37)$ & 0.141 \\
\hline
\end{tabular}

$\mathrm{BMI}$, body mass index; $\mathrm{Cl}$, confidential interval; eGFR, estimated glomerular filtration rate by MDRD (Modification of Diet in Renal Disease Study) equation; HR, hazard ratio.

Table 4. Incidence of catheter removal over the entire observation period

\begin{tabular}{lccc}
\hline & $\begin{array}{c}\text { Percutaneous } \\
\text { group }(n=103)\end{array}$ & $\begin{array}{c}\text { Surgical } \\
\text { group }(n=74)\end{array}$ & P value \\
\hline Catheter removal & $63(61.2)$ & $38(51.4)$ & \\
Catheter failure & $23(22.3)$ & $15(20.3)$ & 0.742 \\
$\quad$ Infectious complication & $17(16.5)$ & $11(14.9)$ & \\
Mechanical complication & $6(5.8)$ & $4(5.4)$ & \\
Kidney transplantation & $28(27.2)$ & $18(24.3)$ & 0.669 \\
Death & $12(11.7)$ & $5(6.8)$ & 0.726 \\
\hline
\end{tabular}

Data are presented as number (\%).

sion analysis for variables associated with catheter failure. In univariate and multivariate analyses, the PD catheter insertion method was not associated with catheter failure (Table 3).

The incidence of catheter failure during the entire observation period was $22.3 \%(23 / 103)$ in the percutaneous group and $20.3 \%(15 / 74)$ in the surgical group $(P=$ NS; Table 4). The incidence of catheter removal within 1 year was $3.9 \%(4 / 103)$ in the percutaneous group and $2.7 \%(2 / 74)$ in the surgical group $(P=$ NS). One refractory peritonitis, two pseudomembranous colitis (PMC), and one refractory tunnel infection resulted in catheter removal in the percutaneous group. One ultrafiltration failure and one refractory peritonitis were the causes of catheter removal in the surgical group. During the observation period, the incidence of death was $12 / 103$ (11.7\%) in the percutaneous group and 5/74 (6.8\%) in the surgical group $(P=$ NS; Table 4$)$. The incidence of transplantation was $28 / 103(27.2 \%)$ in the percutaneous group and 18/74 (24.3\%) in the surgical group ( $P=$ NS; Table 4$)$.

\section{Discussion}

In this study, the percutaneous group using a modified Seldinger percutaneous insertion method and immediate initiation of PD with no break-in period showed similar 90-day infectious and mechanical complication rates compared with the surgical group that had a longer break-in period after catheter insertion. During the entire observation period, the infectious and mechanical complication-free survival did not show any significant differences between the two groups. Moreover, in the noninferiority test, the percutaneous method was not inferior to the surgical method for catheter survival.

ESRD patients experience longer hospitalization and greater morbidity compared to patients with normal renal function $[6,16]$. Higher BUN was regarded as a risk factor for surgical outcomes even in elective surgery [17]. In this study, the percutaneous group showed higher BUN level than the surgical group; nevertheless, this was not associated with an increased rate of infection or bleeding. The percutaneous group did not show increased early complications, suggesting benefit from little or no dissection of the rectus muscle.

Increased pressure within the abdominal cavity could cause pericatheter leakage or hernia. The leakage appeared more frequently in continuous ambulatory $\mathrm{PD}$ (CAPD) rather than automated PD (APD) [18]. For uremic 
patients not requiring emergent dialysis, immediate initiation of PD after catheter insertion is needed for avoiding CVC-related complications; however, the initiation of CAPD should be considered against the potential risk of early pericatheter leakage or hernia [11]. In our protocol, the start of CAPD with $500 \mathrm{~mL}$ per change and gradual increase of dwelling volume was done. Immediate initiation of CAPD after catheter insertion was not accompanied with increased pericatheter leakage or hernia. Low incidence of leakage could be the result from definite positioning of the deep cuff within the rectus muscle and purse-string sutures around the deep cuff using PDS suture material [19], instead of previously used silk and braided suture material to minimize possible inflammation in the percutaneous group.

As shown in our study, the surgical group also had a low incidence of pericatheter leakage and peritonitis compared with a previously published study [12]. This could be explained by the restraint of physical activity by maintaining a supine position for three postoperative days that was applied to the surgical group. Although a breakin period shorter than two weeks was applied, delayed initiation of PD could also have time to anchor and heal around the deep cuff to a certain extent.

Late referred ESRD patients tend to have a longer hospital stay than fully planned patients [20]. In our study of urgent-start PD, the length of hospital stay in the percutaneous group was somewhat longer, but the incidence of bridging HD was similar in both groups. Most of the percutaneous group stayed in the hospital until infusing full volume dialysate, with observation of any complications or discomfort. In contrast, delayed initiation of PD in the surgical group might influence the early discharge without reaching $2 \mathrm{~L}$ dwelling to avoid a long hospital stay.

In the study of Wallace et al [21], about $60 \%$ of PD catheter placement was done by general surgeons. Nephrologists, interventional radiologists, and vascular surgeons were available at $20 \%$ of the study sites. Asif et al [22] demonstrated an improvement in PD utilization with a program for catheter insertion by nephrologists. Several other reports showed favorable results by nephrologists $[14,23]$. Our study showed no significant differences in outcomes between operation by nephrologists or by surgeons. For urgent-start PD requiring 'quick decisionmaking' for improving uremic complications, percutaneous catheter insertion by nephrologists and immediate start with no break-in period could be a good viable option.

There are several limitations to this study. This is a nonrandomized study with heterogeneity in the same group such as mixed operation method and no fixed initiation of PD protocol in the surgical group in comparison with the method in the percutaneous group. For defining the urgent-start PD group, the initial presentation of uremic symptoms and subjective urgency to dialysis were not collected due to limited medical records.

In conclusion, this study suggests that urgent-start PD can be safely applied with percutaneous PD catheter insertion with no break-in period by nephrologists. We recommend a protocol of shorter duration for rapid increase in the amount of PD solution after percutaneous PD catheter insertion and are looking forward to more favorable data for percutaneous PD catheter insertion in the urgent setting.

\section{Conflicts of interest}

All authors have no conflicts of interest to declare.

\section{Funding}

This work was supported by a Pusan National University Research Grant, 2018 (201812750001).

\section{Authors' contributions}

Joo Hui Kim and Min Jeong Kim designed/performed the study and wrote the manuscript. Byung-Min Ye and June Hyun Kim provided intellectual content and critically assessed the work. Min Jeong Kim, Hyo Jin Kim, and Miyeun Han interpreted the data. Seorin Kim and Harin Rhee drafted and revised the article. Il Young Kim, Sang Heon Song, Eun Young Seong, and Soo Bong Lee analyzed and interpreted data and drafted and revised the article. Dong Won Lee revised the article and approved the final version for publication.

\section{References}

[1] Foley RN, Chen SC, Collins AJ. Hemodialysis access at initiation in the United States, 2005 to 2007: still "catheter first". Hemodial Int 2009;13:533-542. 
[2] Blake PG, Finkelstein FO. Why is the proportion of patients doing peritoneal dialysis declining in North America? Perit Dial Int 2001;21:107-114.

[3] Ishani A, Collins AJ, Herzog CA, Foley RN. Septicemia, access and cardiovascular disease in dialysis patients: the USRDS Wave 2 study. Kidney Int 2005;68:311-318.

[4] Koch M, Kohnle M, Trapp R, Haastert B, Rump LC, Aker S. Comparable outcome of acute unplanned peritoneal dialysis and haemodialysis. Nephrol Dial Transplant 2012;27:375380.

[5] Ourives AP, Gonçalves SS, Siqueira RA, et al. High rate of Candida deep-seated infection in patients under chronic hemodialysis with extended central venous catheter use. Rev Iberoam Micol 2016;33:100-103.

[6] Yoon HB, Park HC, Lee H, et al. Treatment outcomes and prognostic factors for peritoneal dialysis patients based on single center experience over 18 years. Kidney Res Clin Pract 2009;28:19-31.

[7] Kim H, Ryu DR. A prime determinant in selecting dialysis modality: peritoneal dialysis patient survival. Kidney Res Clin Pract 2017;36:22-28.

[8] Povlsen JV, Ivarsen P. How to start the late referred ESRD patient urgently on chronic APD. Nephrol Dial Transplant 2006;21 Suppl 2:ii56-ii59.

[9] Ghaffari A. Urgent-start peritoneal dialysis: a quality improvement report. Am J Kidney Dis 2012;59:400-408.

[10] Dombros N, Dratwa M, Feriani M, et al. European best practice guidelines for peritoneal dialysis. 3 Peritoneal access. Nephrol Dial Transplant 2005;20 Suppl 9:ix8-ix12.

[11] Figueiredo A, Goh BL, Jenkins S, et al. Clinical practice guidelines for peritoneal access. Perit Dial Int 2010;30:424429.

[12] Yang YF, Wang HJ, Yeh CC, Lin HH, Huang CC. Early initiation of continuous ambulatory peritoneal dialysis in patients undergoing surgical implantation of Tenckhoff catheters. Perit Dial Int 2011;31:551-557.

[13] Alkatheeri AM, Blake PG, Gray D, Jain AK. Success of urgent-start peritoneal dialysis in a large Canadian renal program. Perit Dial Int 2016;36:171-176.

[14] Jo YI, Shin SK, Lee JH, Song JO, Park JH. Immediate initiation of CAPD following percutaneous catheter placement without break-in procedure. Perit Dial Int 2007;27:179-183.

[15] Li PK, Szeto CC, Piraino B, et al. ISPD peritonitis recommendations: 2016 update on prevention and treatment. Perit Dial Int 2016;36:481-508.

[16] Schneider CR, Cobb W, Patel S, Cull D, Anna C, Roettger R. Elective surgery in patients with end stage renal disease: what's the risk? Am Surg 2009;75:790-793.

[17] Abe H, Mafune K. Risk factors for maintenance hemodialysis patients undergoing elective and emergency abdominal surgery. Surg Today 2014;44:1906-1911.

[18] Del Peso G, Bajo MA, Costero O, et al. Risk factors for abdominal wall complications in peritoneal dialysis patients. Perit Dial Int 2003;23:249-254.

[19] Willatt DJ, Durham L, Ramadan MF, Bark-Jones N. A prospective randomized trial of suture material in aural wound closure. J Laryngol Otol 1988;102:788-790.

[20] Dogan E, Erkoc R, Sayarlioglu H, Durmus A, Topal C. Effects of late referral to a nephrologist in patients with chronic renal failure. Nephrology (Carlton) 2005;10:516-519.

[21] Wallace EL, Fissell RB, Golper TA, et al. Catheter insertion and perioperative practices within the ISPD North American Research Consortium. Perit Dial Int 2016;36:382-386.

[22] Asif A, Pflederer TA, Vieira CF, Diego J, Roth D, Agarwal A. Does catheter insertion by nephrologists improve peritoneal dialysis utilization? A multicenter analysis. Semin Dial 2005;18:157-160.

[23] Al-Hwiesh AK. Percutaneous peritoneal dialysis catheter insertion by a nephrologist: a new, simple, and safe technique. Perit Dial Int 2014;34:204-211. 